\title{
Exploring Driver Injury Severity in Single-Vehicle Crashes under Foggy Weather and Clear Weather
}

\author{
Fulu Wei, ${ }^{1,2}$ Zhenggan Cai, ${ }^{1}$ Pan Liu, ${ }^{2}$ Yongqing Guo ${ }^{D},{ }^{1} X$ in Li, ${ }^{1}$ and Qingyin $\mathrm{Li}^{1}$ \\ ${ }^{1}$ Department of Transportation Engineering, Shandong University of Technology, Zibo 255000, China \\ ${ }^{2}$ Department of Transportation Planning and Management, Southeast University, Nanjing 210096, China \\ Correspondence should be addressed to Yongqing Guo; yongqing.guo@sdut.edu.cn
}

Received 11 March 2021; Accepted 19 July 2021; Published 11 August 2021

Academic Editor: Jinjun Tang

Copyright (c) 2021 Fulu Wei et al. This is an open access article distributed under the Creative Commons Attribution License, which permits unrestricted use, distribution, and reproduction in any medium, provided the original work is properly cited.

The purpose of this study is to investigate and compare the significant influencing factors of driver injury severity in single-vehicle (SV) crashes under foggy and clear weather conditions. Based on data for SV crashes in Shandong Province, China, the mixed logit model (MLM) was employed to interpret driver injury severity for SV crashes in clear and foggy weather. The results showed that there are significant differences in the influencing factors of the severity of SV crashes in foggy and clear weather. Specifically, 15 factors are significantly associated with the severity of SV crashes in clear weather, and 18 factors are significantly associated with the severity of SV crashes in foggy weather. In addition, young drivers (age $<30$ ), non-dry road surfaces, and signal control significantly influence the severity of foggy weather crashes but not clear weather crashes. Self-employment and weekends have significant effects on the severity of crashes only in clear weather. Interestingly, drivers whose occupation is farming showed opposite trends in the effect of crash severity in foggy and clear weather. Based on the findings of this research, some potential countermeasures can be adopted to reduce crash severity in foggy and clear weather.

\section{Introduction}

The investigation of single-vehicle (SV) crash severity is of great interest to numerous transportation experts due to its high fatality rate. According to statistics from the National Highway Traffic Safety Administration (NHTSA) in 2017, SV crashes and fatal SV crashes accounted for 30\% and 54\% of total collisions and fatalities, respectively [1], and the fatality rate of drivers was higher than for other types of traffic collisions [2]. In Singapore, for example, the risk of fatality for drivers in SV crashes is 1.7 times compared to two-vehicle crashes [3]. Such information shows that extremely adverse effects are initiated by SV crashes; to better understand the impact of contributing factors on the severity of SV crashes, in-depth research is urgently needed.

Some differences in SV crashes between foggy and clear weather may exist $[4,5]$. The low visibility caused by fog affects driver behavior and the driving environment, which can lead to contributing effects on traffic collisions that are different from those in clear weather. Foggy weather and clear weather effects in terms of both crash risk and severity are developed [6]. To clarify the influencing factors of crash risk, Wu et al. [7] compared the variability of traffic patterns and crash risk between clear and foggy weather. The research showed that traffic volume and vehicle speed on foggy days tend to decrease compared to sunny days. In addition, the risk of crashes on foggy days is significantly increased near ramps or with large traffic flow. However, to date, the difference in contributing factors influencing the severity of SV crashes between foggy and clear days has not been studied. $\mathrm{Li}$ et al. [8] discussed the contributing factors of low visibilityrelated SV crash severity, but the exploration did not include the variability of influencing factors in foggy and clear weather. Considering the different characteristics of traffic flow in different regions, the research conclusion according to US collision data cannot be directly transferred to other countries. For example, Feng et al. [9] uses the crash data of Shanghai, China, and Florida, USA, to establish safety performance functions (SPFs), respectively, and investigate the transferability between the two SPFs. It was found that 
the transferability of SPFs between Florida and the Chinese cities turned out to be unsatisfactory and this phenomenon was contributed by the difference in traffic flow.

Based on an in-depth literature review of the potential correlation between different collision models, the existing literature on weather-related crashes can provide reference for modeling clear and fog-related SV crashes. In the existing literature, foggy and clear weather are usually analyzed and modeled as categorical variables of weather factors. For example, Wen and Xue [10] classified weather factors into four variables-sunny, rainfall, snowfall, and foggy. The study found that compared with sunny weather, the probability of fatal accidents increased by $3.4 \%, 3.3 \%$, and $15.7 \%$, respectively, for rainfall, snowfall, and fog. Research on the influence of severe weather on SV crashes mainly focuses on rainfall or snowfall weather. For example, Li et al. [11] developed a mixed logit model (MLM) for SV crashes in rainy weather to identify the influence of different factors on crash severity. Yu et al. [12] studied SV crashes in snowy weather and found that male drivers had individual heterogeneity on the impact of crash severity. In research on fog-related collisions, Moore and Cooper [13] conducted a study of traffic collisions in foggy weather and found that fog increases the probability of traffic collisions by $16 \%$, noting that fog is one of the most feared weather hazards for drivers. Abdel-Aty et al. [14] developed a multilevel ordered logit model using collision data from Florida to study fog-related crashes. Early winter mornings and nights without streetlights were found to be the highest crash periods. It was considered that fog would dilute color depth and further aggravate the severity of traffic collisions. Later, based on the fact that fog can affect a driver's correct judgment of driving distance, Tarel et al. [15] proposed an algorithm to improve the visibility of road images, which was used to improve existing advanced driver assistance systems (ADAS) and improve traffic safety in foggy weather.

Modeling approaches are recognized as an important part of traffic safety research, and many discrete choice models have been established to analyze SV crashes [16]. Among them, the multinomial logit model (MNL) has been widely used. However, MNL involves two limitations: (1) it is based on the assumption of independence of irrelevant alternatives (IIA); this hypothesis is not always accepted as the model can be influenced by unobserved factors [17]; and, (2) the parameter is treated as a fixed value, which cannot reflect the individual heterogeneity across observations; this may lead to bias in parameter estimation. (a comprehensive analysis of the heterogeneous effects of independent variables was conducted by Mannering et al. [18]).

To overcome the limitation of traditional econometric models, the method of using an MLM to analyze the severity of SV crashes was proposed. MLM allows parameters to vary randomly across observations, which obviates the limitations of MNL. The diversity of random distribution forms makes MLM highly flexible and adaptable. Ideally, MLM can fit all data samples with random effects. Currently, most studies have concluded that the fit performance of MLM for crash data is significantly better than that of traditional discrete choice models. For instance, Ye and Lord [19] verified that the fit performance of MLM outperforms conventional models that do not account for heterogeneity by allowing the model parameters to vary across the observations according to a predefined distribution. Therefore, the MLM was established to identify the influence of contributing factors on SV crash severity in foggy and clear weather. The marginal effects of significant independent variables in MLM were calculated to further quantify the effect of each contributing factor on crash severity.

In addition, considering that traffic crashes may lead to multiple casualties, three methods are generally used to classify the severity of crashes: (1) based on the severity of the driver's injury; (2) based on the most seriously injured passengers; and, (3) based on the most serious injury in the crash [20-22]. It has been widely recognized that there are differences existing in safety precautions among passengers as well as between drivers and passengers. The probability of injury is often different. To reduce the influence of potential factors on crash severity, driver injury severity is used as the index to measure crash severity.

The main objective of this research was to investigate the contributing factors of SV crash severity in foggy and clear weather. More specifically, this study focused on answering the following two questions: (1) is the prediction effect of MLM on the severity of SV crashes in foggy weather and clear weather satisfactory? (2) What are the different effects of different factors on the severity of SV crashes in foggy and clear weather?

\section{Data Collection}

With support of the Shandong Department of Transportation (SDOT), data on SV crashes over the five-year period of 2015-2019 that occurred on the national highway in winter were collected as the sample database. Considering that weather and visibility information were by observation, data were estimated by traffic police at the scenes of accident, and some errors may exist. According to the time of crashes, these data were obtained from the Shandong Climatic Data Center (SCDC).

National highways in China are indispensable and carry most intercity traffic volumes. The grade of the national highway is very high, and the average speed is fast. As a result, vehicles operating on national highways are more prone to serious crashes. Furthermore, in Shandong, the alternate occurrence of foggy and clear days often occurs in winter; thus, it is more suitable to use winter for studying the difference of crashes between foggy and clear weather.

In this study, visibility below $200 \mathrm{~m}$ was treated as foggy weather. Therefore, fog-related SV crashes were extracted when the visibility was less than $200 \mathrm{~m}$. Visibility above $200 \mathrm{~m}$ was considered as clear weather. It is noted that rainfall and snowfall may also lead to low visibility; therefore, this part of the data was eliminated.

Based on the above selection rules, raw data for 11,126 SV crashes were obtained by data extraction. Of these, outof-control crashes and rollover crashes were excluded due to the them accounting for less than 1\%. An additional 407 (3.9\%) crash records were excluded due to missing vital 
information. Ultimately, a total of 10,137 crashes were employed for subsequent analysis, including 5,138 SV crashes in foggy weather (SVCF) and 4,999 SV crashes in clear weather (SVCC). Crash severity was based on the severity of driver injuries and was classified as no injury, slight injury, and serious or fatality injury (a driver passing away within seven days was regarded as a fatal event). In the dataset, there were 6,854 (67.6\%) no-injury crashes, 2,051 (20.2\%) slight-injury crashes, and 1,232 (12.2\%) serious or fatality injury (SFI) crashes. Annual distribution statistics of crashes with different severities are shown in Figure 1. As shown, the number of crashes decreased with the increase of injury severity, which is in line with reality.

Fifteen factors were chosen as independent variables from driver characteristics, vehicle type, road characteristics, and other characteristics. All independent variables have been discredited for modeling. More specifically, snowy pavement and wet pavement were combined as the non-dry road surface; therefore, dry and non-dry road surfaces were contained in road condition. In addition, more than 20 crash types were contained in the original records, which were combined into four categories: collision with non-fixed object, collision with fixed object, collision with pedestrian, and other crashes. There were nearly 80 types of motor vehicles. In the preprocessing process, less than $1 \%$ of vehicle types (e.g., farm machinery and large buses) were classified into other vehicle types. Ultimately, vehicle types were categorized into five types: passenger car, motorcycle, pickup, truck, and other vehicle types. The specific variable classification and description are shown in Table 1. Considering that the actual distribution of factors in the sample was masked by the discrete variables, the number changes of the two archetypal continuously distributed variables, driver age, and time of crashes are shown in Figures 2 and 3.

From Table 1, we can see that the percentage of SVCF resulting in slight injuries and SFI are $22.0 \%$ and $14.7 \%$, respectively, which are higher than the percentage of SVCC $(18.3 \%$ and 9.7\%). This indicates a significant difference in the driver injury severity between fog-related SV crashes and clear-related SV crashes. For different age groups, the number of crashes is roughly the same in both foggy weather and clear weather. Figure 2 shows the trend more clearly; the highest percentage is between ages 38 and 47 in both weather conditions. In addition, it can be seen from Figure 3 that a significant differences' trend is shown about time characteristics during clear and foggy weather. The number of crashes in clear weather shows a slow upward trend from $06: 00 \mathrm{am}-18: 00 \mathrm{pm}$, and the peak value is at $1500 \mathrm{pm}-1800 \mathrm{pm}$. The number of crashes in foggy weather shows an upward trend from 15:00 pm-21:00 pm, and the peak value is at $18: 00 \mathrm{pm}-21: 00 \mathrm{pm}$. The peak value of crashes in foggy weather is significantly higher than that in clear weather. These data further exemplify the differences in traffic operating characteristics between foggy and clear conditions. It is necessary to study the differences between SVCC and SVCF.

\section{Methodology}

3.1. Mixed Logit Model. The mixed logit probability can be derived from utility-maximizing behavior. Assume that driver injury severities are divided into $J$ categories (in this

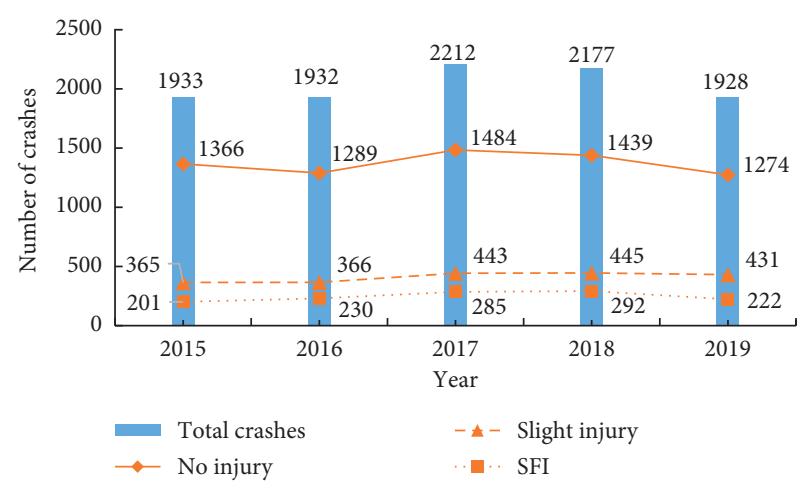

FIGURE 1: Annual distribution of number of crashes with different severities.

research, $J=3$ ). The utility function of the $i$ th crash with the injury severity $j$ can be specified as the following equation:

$$
U_{i j}=x_{i j}^{\prime} \beta_{j}+\varepsilon_{i j}, \quad(i=1, \ldots, n ; j=1, \ldots, J),
$$

where $U_{i j}$ is the utility when the severity of the $i$ th crash is $j$, $x_{i j}^{\prime}$ represents the independent variables, $\varepsilon_{i j}$ is the observed disturbance term, $\beta_{j}$ is the coefficient of the independent variable, and $n$ indicates sample size.

When the injury severity of the $i$ th crash is $j$, the utility is higher than that of other severe categories. Assuming that $\varepsilon_{i j}$ obeys the generalized extreme value distribution, the probability of injury severity $j$ for the $i$ th crash can be expressed as equation (2), and it is also known as the MNL model:

$$
P\left(y_{i}=j \mid x_{i j}\right)=P\left(U_{i j} \geq U_{i k}, \forall k \neq j\right)=\frac{\exp \left(x_{i}^{\prime} \beta_{j}\right)}{\sum_{j=1}^{J} \exp \left(x_{i}^{\prime} \beta_{j}\right)} .
$$

Considering the random distribution of parameter across observations, the MLM can be expressed as the following equation:

$$
P\left(y_{i}=j \mid \varphi\right)=\int \frac{\exp \left(x_{i}^{\prime} \beta_{j}\right)}{\sum_{j=1}^{J} \exp \left(x_{i}^{\prime} \beta_{j}\right)} f\left(\beta_{j} \mid \varphi\right) d \beta_{j},
$$

where $f\left(\beta_{j} \mid \varphi\right)$ represents the probability density function of parameter $\beta_{j}, \varphi$ represents an unknown characteristic parameter of the probability density function, such as the mean value $\mu$ and the variance $\sigma$ of normal distribution, which can be expressed as $\varphi=\{\mu, \sigma\}$, and parameter $\beta_{j}$ varies across observations which may be random or fixed. MLM will degrade to MNL when all parameters in the model are fixed.

3.2. Marginal Effects. Given that the coefficient in MLM cannot measure the quantitative relationship between the independent variable and the dependent variable, the marginal effects of significant independent variables in the model were calculated. The mathematical meaning of the marginal effect refers to the probability change of a certain crash severity when a variable changes by one unit, while the 
TABLE 1: Classification statistics of variables.

\begin{tabular}{|c|c|c|c|c|c|c|}
\hline \multirow{2}{*}{ Variables } & \multicolumn{2}{|c|}{ SVCF } & \multicolumn{2}{|c|}{ SVCC } & \multicolumn{2}{|c|}{ Total } \\
\hline & Number & Rate (\%) & Number & Rate (\%) & Number & Rate (\%) \\
\hline Number of crashes & 5138 & 50.7 & 4999 & 49.3 & 10137 & 100 \\
\hline \multicolumn{7}{|l|}{ Severity } \\
\hline No injury* & 3253 & 63.3 & 3601 & 72.0 & 6854 & 67.6 \\
\hline Slight injury & 1135 & 22.0 & 916 & 18.3 & 2051 & 20.2 \\
\hline SFI & 750 & 14.7 & 482 & 9.7 & 1232 & 12.2 \\
\hline \multicolumn{7}{|l|}{ Driver characteristics } \\
\hline \multicolumn{7}{|l|}{ Driver gender } \\
\hline Male & 4860 & 94.5 & 4622 & 92.4 & 9482 & 93.5 \\
\hline Female* & 278 & 5.5 & 377 & 7.6 & 655 & 6.5 \\
\hline \multicolumn{7}{|l|}{ Driver age } \\
\hline$<30$ & 1195 & 23.3 & 1095 & 21.9 & 2290 & 22.6 \\
\hline $30-60^{*}$ & 3658 & 71.2 & 3588 & 71.8 & 7246 & 71.5 \\
\hline$>60$ & 285 & 5.5 & 316 & 6.3 & 601 & 5.9 \\
\hline \multicolumn{7}{|l|}{ Seat belt used } \\
\hline Not used & 1211 & 23.6 & 875 & 17.6 & 2086 & 20.6 \\
\hline Used $^{*}$ & 3927 & 76.4 & 4124 & 82.4 & 8051 & 79.4 \\
\hline \multicolumn{7}{|l|}{ Alcohol-impaired } \\
\hline $\mathrm{No}^{*}$ & 4552 & 82.6 & 4264 & 85.3 & 8816 & 83.9 \\
\hline Yes & 586 & 11.4 & 735 & 14.7 & 1321 & 13.1 \\
\hline \multicolumn{7}{|l|}{ Career } \\
\hline Staff* & 594 & 11.5 & 809 & 16.1 & 1403 & 13.8 \\
\hline Self-employed & 768 & 14.9 & 1042 & 20.8 & 1810 & 17.8 \\
\hline Farmer & 3296 & 64.1 & 2622 & 52.4 & 5918 & 58.3 \\
\hline Others & 480 & 9.3 & 526 & 10.5 & 1006 & 9.9 \\
\hline \multicolumn{7}{|l|}{ Driving experience } \\
\hline$<4$ years & 983 & $19.1 \%$ & 1031 & $20.6 \%$ & 2048 & $20.2 \%$ \\
\hline 4-10 years* & 2026 & $39.4 \%$ & 1871 & $37.4 \%$ & 3943 & $38.9 \%$ \\
\hline$>10$ years & 2125 & $41.4 \%$ & 1977 & $39.5 \%$ & 4146 & $40.9 \%$ \\
\hline \multicolumn{7}{|l|}{ Driving license } \\
\hline With license & 4530 & $88.1 \%$ & 4484 & $89.6 \%$ & 9014 & $89.0 \%$ \\
\hline Without license* & 608 & $11.9 \%$ & 515 & $10.3 \%$ & 1123 & $11.0 \%$ \\
\hline \multicolumn{7}{|l|}{ Vehicle characteristics } \\
\hline \multicolumn{7}{|l|}{ Vehicle type } \\
\hline Passenger car* & 1859 & $36.1 \%$ & 2019 & $40.3 \%$ & 3878 & $38.2 \%$ \\
\hline Motorcycle & 1642 & $31.9 \%$ & 1101 & $22.0 \%$ & 2743 & $27.0 \%$ \\
\hline Pickup & 351 & $6.8 \%$ & 439 & $8.7 \%$ & 790 & $7.7 \%$ \\
\hline Truck & 1114 & $21.6 \%$ & 1194 & $23.8 \%$ & 2308 & $22.7 \%$ \\
\hline Other & 172 & $3.3 \%$ & 246 & $4.9 \%$ & 418 & $4.1 \%$ \\
\hline \multicolumn{7}{|l|}{ Road characteristics } \\
\hline Road surface & & & & & & \\
\hline Non-dry & 526 & $10.2 \%$ & 263 & $5.3 \%$ & 789 & $7.8 \%$ \\
\hline Dry* & 4612 & $89.7 \%$ & 4736 & $94.7 \%$ & 9348 & $92.2 \%$ \\
\hline Other characteristics & & & & & & \\
\hline Traffic controls & & & & & & \\
\hline No control* & 1684 & $32.7 \%$ & 1516 & $30.3 \%$ & 3200 & $31.5 \%$ \\
\hline Signal control & 205 & $3.9 \%$ & 292 & $5.8 \%$ & 497 & $4.9 \%$ \\
\hline Stop-yield sign & 2899 & $56.4 \%$ & 2964 & $59.2 \%$ & 5863 & $57.8 \%$ \\
\hline Other control methods & 350 & $6.8 \%$ & 227 & $4.5 \%$ & 577 & $5.8 \%$ \\
\hline Week & & & & & & \\
\hline Monday or Friday & 2216 & $43.1 \%$ & 1394 & $27.8 \%$ & 2867 & $28.2 \%$ \\
\hline Tuesday-Thursday* & 1473 & $28.6 \%$ & 2213 & $44.2 \%$ & 4429 & $43.6 \%$ \\
\hline Weekend & 1449 & $28.2 \%$ & 1392 & $27.8 \%$ & 2841 & $28.0 \%$ \\
\hline Intersection & & & & & & \\
\hline $\mathrm{No}^{*}$ & 3981 & $77.4 \%$ & 3563 & $71.2 \%$ & 7544 & $74.4 \%$ \\
\hline Yes & 1157 & $22.6 \%$ & 1436 & $28.7 \%$ & 2593 & 25.5 \\
\hline Time of accident & & & & & & \\
\hline $00: 00-07: 00$ & 1068 & $20.7 \%$ & 442 & $8.8 \%$ & 1510 & $14.8 \%$ \\
\hline $07: 00-09: 00$ & 299 & $5.8 \%$ & 1056 & $21.1 \%$ & 1355 & $13.3 \%$ \\
\hline
\end{tabular}


TABLE 1: Continued.

\begin{tabular}{|c|c|c|c|c|c|c|}
\hline \multirow{2}{*}{ Variables } & \multicolumn{2}{|c|}{ SVCF } & \multicolumn{2}{|c|}{ SVCC } & \multicolumn{2}{|c|}{ Total } \\
\hline & Number & Rate (\%) & Number & Rate $(\%)$ & Number & Rate (\%) \\
\hline $09: 00-17: 00^{*}$ & 802 & $15.6 \%$ & 2613 & $52.2 \%$ & 3415 & $33.6 \%$ \\
\hline $17: 00-20: 00$ & 1587 & $30.8 \%$ & 664 & $13.2 \%$ & 2251 & $22.2 \%$ \\
\hline $20: 00-24: 00$ & 1382 & $26.8 \%$ & 224 & $4.4 \%$ & 1606 & $15.8 \%$ \\
\hline \multicolumn{7}{|l|}{ Area } \\
\hline Urban* & 3011 & $58.6 \%$ & 3494 & $69.8 \%$ & 6505 & $64.2 \%$ \\
\hline Rural & 2127 & $41.3 \%$ & 1505 & $30.2 \%$ & 3632 & $35.8 \%$ \\
\hline \multicolumn{7}{|l|}{ Crash type } \\
\hline Non-fixed object* & 3670 & $71.4 \%$ & 4234 & $84.6 \%$ & 7904 & $77.9 \%$ \\
\hline Fixed object & 334 & $6.5 \%$ & 195 & $3.9 \%$ & 529 & $5.2 \%$ \\
\hline Collision with pedestrian & 1079 & $21.0 \%$ & 500 & $10.0 \%$ & 1579 & $15.5 \%$ \\
\hline Other object & 55 & $1.1 \%$ & 70 & $1.4 \%$ & 125 & $1.2 \%$ \\
\hline
\end{tabular}

Note: ${ }^{*}$ indicates that that variable was a reference variable in the model.

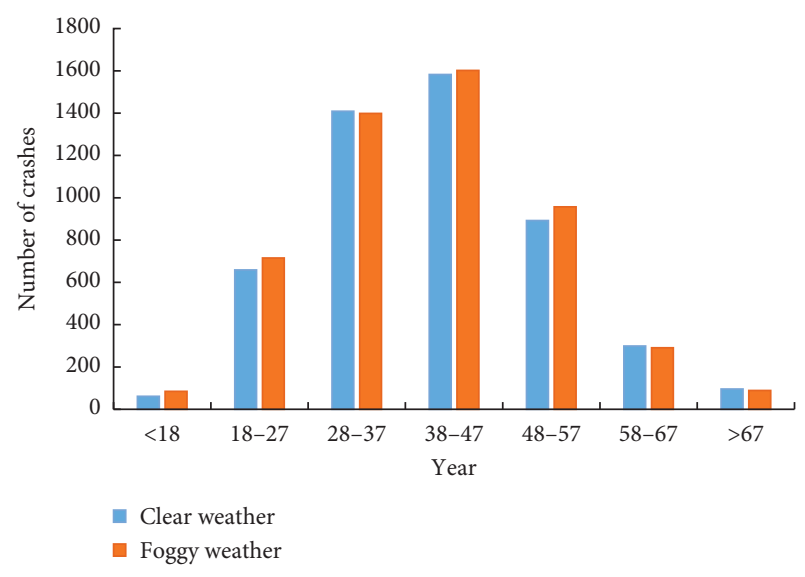

Figure 2: Driver age distribution characteristics of crashes.

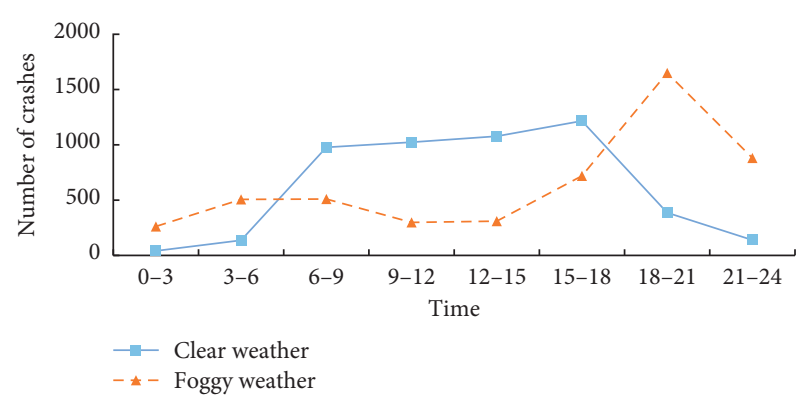

Figure 3: Time distribution characteristics of crashes.

value of other variables remains unchanged. Since the variables in the model have been transformed into discrete variables (the variables' value is 0 or 1 ), the marginal effects of the variables are shown in the following equation:

$$
E_{x_{i j k}}^{P\left(y_{i}=j\right)}=P\left(y_{i}=j \mid x_{i j k}=1\right)-P\left(y_{i}=j \mid x_{i j k}=0\right),
$$

where $E_{x_{i j k}}^{P\left(y_{i}=j\right)}$ denotes the marginal effect of the $k$ th significant independent variable when the injury severity of the $i$ th crash is $j$, it can measure the probability of the injury level $j$ when the value of the binary variable $x_{k}$ changes (from 0 to 1 or from 1 to 0$), x_{i j k}$ represents the $k$ th significant independent variable, and $P\left(y_{i}=j \mid x_{i j k}=1\right)$ denotes the probability when the severity of the $i$ th crash is $j$ given the value of independent variable $x_{i j k}$. For variables with random effects in the sample, the mean of the model coefficient is used to calculate the marginal effect of the variable.

3.3. Model Diagnostics. Model diagnostics mainly include tests for validity and goodness-of-fit. The validity test is achieved by checking the likelihood ratio (also known as the chi-square test). The likelihood ratio test is calculated, as shown in the following equation:

$$
\chi^{2}=-2\left(\operatorname{LL}(0)-\operatorname{LL}\left(\beta_{j}\right)\right) \sim \chi^{2}(k),
$$

where the likelihood ratio test for the model is denoted by $\chi^{2}$, LL(0) denotes log-likelihood at constants (the intercept model includes only the constant term), the log-likelihood at convergence is denoted by $\operatorname{LL}\left(\beta_{j}\right)$, and $k$ represents the number of model parameters (the full model with the constant term and all predicting variables). When the value of $\chi^{2}$ is greater than the value of $\chi^{2}(k)$ on the given significance level $\alpha$, the test is satisfied.

In terms of goodness-of-fit evaluation, the Akaike Information Criterion (AIC) avoids overfitting by introducing a penalty term for complexity. Thus, a criterion for weighing complexity and fit satisfaction was provided. However, the penalty factor for the parameters' number in AIC is not affected by the observation size (the penalty factor is always 2). The weakness of AIC will cause the estimated value to deviate from the real value, when the sample size is large enough. On the contrary, the Bayesian Information Criterion (BIC) compensates for the deficiency of AIC by introducing sample parameters, which makes the penalty term higher than AIC. Hence, the BIC can provide more reliable estimation.

Another evaluation index of model fitting is the $\mathrm{McF}$ adden pseudo $R$-squared. The ratio of the log-likelihood at convergence and the log-likelihood at constants measures the level of improvements over the constant term model offered by the full model. Satisfactory fit will be reflected by the larger McFadden pseudo $R$-squared value. Therefore, the MLM was diagnosed by AIC, BIC, as well as McFadden 
pseudo $R$-squared. The formulas for AIC, BIC, and McFadden pseudo $R$-squared are shown in the following equations:

$$
\begin{aligned}
\mathrm{AIC} & =2 k-2 \operatorname{LL}\left(\beta_{j}\right), \\
\mathrm{BIC} & =k \ln (n)-2 \operatorname{LL}\left(\beta_{j}\right), \\
\text { McFadden Pseudo } R^{2} & =1-\frac{\operatorname{LL}\left(\beta_{j}\right)}{\operatorname{LL}(0)},
\end{aligned}
$$

where $k$ is the number of parameters and $n$ is the number of samples in the dataset. In general, the smaller the value of AIC and BIC, the better the fitting of the model to the dataset.

3.4. Model Transferability. The crash severity models may be different between clear weather and foggy weather conditions. It is necessary to investigate the transferability of the crash model under different scenarios. The transferability assessment of the crash model can be achieved by calculating the transfer index (TI), which is calculated as follows:

$$
\operatorname{TI}_{b}\left(\beta_{a}\right)=\frac{\operatorname{LL}_{b}\left(\beta_{a}\right)-\mathrm{LL}_{b}\left(\beta_{\text {reference }, b}\right)}{\operatorname{LL}_{b}\left(\beta_{b}\right)-\mathrm{LL}_{b}\left(\beta_{\text {reference }, b}\right)},
$$

where $\mathrm{TI}_{b}\left(\beta_{a}\right)$ means the TI value, when the weather condition is $a$, the accident model with parameter of $\beta_{a}$ is applied to the case of weather condition $b$ and the corresponding TI value, $\mathrm{LL}_{b}\left(\beta_{a}\right)$ denotes the log-likelihood of the model, established by the crash data of weather condition $a$ and being applied to crash data of weather condition $b$, $\mathrm{LL}_{b}\left(\beta_{\text {reference, } b}\right)$ is the log-likelihood of the intercept-only model for weather condition $b$, and $\mathrm{LL}_{b}\left(\beta_{b}\right)$ is the full model built from the crash data of weather condition $b$.

TI criteria have an upper limit of 1 , and no lower limit. The larger the TI value is, the better the transferability of the model. A negative TI value suggests that the transferability of the model is not satisfactory. The intercept-only model which is established under the weather condition $b$ outperforms the full model which is established under the weather condition $a$ in fitting the crash data of weather condition $b$.

\section{Discussion}

Since the probability function in MLM is nonclosed, the analytical solution cannot be achieved by calculating the integral. Therefore, the simulation-based maximum likelihood method was adopted for model estimation. By weighing computational efficiency against the goodness-offit, 1,000 iterations and Halton sequences in simulations were used to ensure effective estimation of parameters [12]. To select the most appropriate form of parameter distribution, it was necessary to test the four commonly-used distributions of the density function (log-normal, uniform, normal, and triangular).
Log-normal distribution requires all parameters to keep the same sign (all positive or all negative). However, the influence of different factors on driver injury severity may be either positively or negatively correlated. Thus, a log-normal distribution was not suitable for this research. Subsequently, the MLM with random coefficients following a log-normal distribution was ignored. When the SVCF model and the SVCC model obey normal distribution, triangular distribution, and uniform distribution, respectively, the fitting results of the models are shown in Table 2. It can be seen from Table 2 that the AIC and BIC of the SVCF model and the SVCC model with normal distribution are slightly better than those of triangular distribution and uniform distribution. The normal distribution provides a more straightforward description about the effect of random variables on injury severity than the triangular and uniform distribution. In this study, all random parameters in MLM were assumed to obey normal distribution to identify the variables that had random influence on injury severity. Normal distribution is also widely used in the existing research.

The MLM was employed to analyze the SVCF and the SVCC. The dependent variables include no injury, slight injury, and SFI. No injury was used as a reference variable during the modeling process. The parameter estimates are shown in Tables 3 and 4, and all parameters given in the model were significant at $90 \%$ confidence level. Average marginal effects are shown in Table 5. In evaluating the fit performance of the crash model, Mujalli and de Oña [23] concluded that a McFadden pseudo $R$-squared value of 0.2 to 0.4 would be sufficient. As can be seen from Tables 3 and 4, the McFadden pseudo $R^{2}$ values for the SVCC and SVCF models were 0.434 and 0.348 , respectively, and the $p$ value for the likelihood ratio test was less than 0.001 . This indicates that the MLM fits the SVCF and SVCC data satisfactorily. Meanwhile, both models contain fixed parameters and random parameters, and the distribution of parameters in different injury severities is different. The results show that the MLM can effectively capture the unobserved heterogeneous across observations. The contributing factors that had a significant impact on injury severity of SVCC and SVCF (especially SFI) are discussed in detail. It should be pointed out that, in the follow-up discussion, the marginal effects were mainly used to quantify the influence of risk factors on the severity of crashes.

4.1. Driver Characteristics. Gender has a significant influence on driver injury severity of both SVCF and SVCC crashes. The marginal effect shows that compared with female drivers, the probability of SFI injury of male drivers in SVCF and SVCC accidents decreased by $4.5 \%$ and $0.3 \%$, respectively [24, 25]. Moreover, the influence of male drivers on the occurrence of SFI crashes in both weather conditions shows a random effect. It can be seen that although the effect of gender on SVCF and SVCC injury severity levels is in the same direction, the proportion of SFI crashes in foggy weather is significantly lower than in clear weather. This finding is very meaningful, which not only can further 
TABLE 2: Fitting index statistics of MLMs under different distributions.

\begin{tabular}{|c|c|c|c|c|c|c|}
\hline \multirow{2}{*}{ Fitting test } & \multicolumn{3}{|c|}{ SVCC model } & \multicolumn{3}{|c|}{ SVCF model } \\
\hline & Normal & Triangular & Uniform & Normal & Triangular & Uniform \\
\hline $\operatorname{LL}\left(\beta_{j}\right)$ & -2187.58 & -2188.19 & -2187.90 & -3029.94 & -3030.51 & -3030.42 \\
\hline AIC & 4491.17 & 4492.39 & 4491.79 & 6159.88 & 6162.15 & 6161.54 \\
\hline BIC & 4869.15 & 4870.34 & 4869.78 & 6487.10 & 6488.41 & 6487.82 \\
\hline
\end{tabular}

TABLe 3: Model estimation results of SVCC.

\begin{tabular}{|c|c|c|c|c|c|c|c|}
\hline \multirow{2}{*}{ Variable } & \multirow{2}{*}{ Description } & \multicolumn{3}{|c|}{ Slight injury } & \multicolumn{3}{|c|}{ SFI } \\
\hline & & Mean & S.E. & $z$-value & Mean & S.E. & $z$-value \\
\hline \multicolumn{8}{|l|}{ Driver characteristics } \\
\hline \multirow{2}{*}{ Gender } & Male & $-0.702^{* *}$ & 0.162 & -4.33 & $-0.491^{*}$ & 0.267 & -1.84 \\
\hline & Std. dev. & - & - & - & 1.241 & 0.108 & - \\
\hline Age & $>60$ & $1.192^{* *}$ & 0.194 & 6.14 & $2.151^{* *}$ & 0.244 & 36.13 \\
\hline Seat belt used & Not used & $0.370^{* *}$ & 0.132 & 2.80 & $2.004^{* *}$ & 0.170 & 11.79 \\
\hline Alcohol-impaired & Yes & $0.950^{* *}$ & 0.183 & 5.19 & $3.580^{* *}$ & 0.194 & 18.45 \\
\hline \multirow{2}{*}{ Career } & Self-employed & - & - & - & $-0.624^{*}$ & 0.265 & -2.35 \\
\hline & Farmer & - & - & - & $-0.450^{*}$ & 0.207 & -2.17 \\
\hline Driving license & With license & $-0.681^{* *}$ & 0.161 & -4.23 & $-0.826^{* *}$ & 0.227 & -3.64 \\
\hline \multicolumn{8}{|l|}{ Vehicle characteristics } \\
\hline \multirow{3}{*}{ Vehicle type } & Motorcycle & $2.594^{* *}$ & 0.143 & 18.14 & $2.442^{* *}$ & 0.226 & 10.81 \\
\hline & Pickup & $-0.638^{* *}$ & 0.167 & -3.82 & $-0.692^{*}$ & 0.318 & -2.18 \\
\hline & Truck & - & - & - & $-0.776^{* *}$ & 0.253 & -3.07 \\
\hline \multicolumn{8}{|l|}{ Other characteristics } \\
\hline \multirow{2}{*}{ Area } & Rural & $0.278^{*}$ & 0.109 & 2.55 & $0.403^{*}$ & 0.166 & 2.43 \\
\hline & Std. dev. & 1.015 & 0.074 & - & - & - & - \\
\hline \multirow[t]{2}{*}{ Week } & Weekend & - & - & - & $0.540^{* *}$ & 0.176 & 3.07 \\
\hline & $07: 00-09: 00$ & $-0.268^{*}$ & 0.127 & -2.111 & - & - & - \\
\hline \multirow[t]{2}{*}{ Time of accident } & $20: 00-24: 00$ & $1.056^{* *}$ & 0.209 & 5.05 & $1.412^{* *}$ & 0.299 & 4.72 \\
\hline & $00: 00-07: 00$ & - & - & - & $0.981^{* *}$ & 0.254 & 3.86 \\
\hline \multirow{2}{*}{ Crash type } & Fixed object & $1.791^{* *}$ & 0.253 & 7.08 & $3.440^{* *}$ & 0.285 & 12.07 \\
\hline & Pedestrian & $-1.997^{* *}$ & 0.216 & -9.25 & $-2.800^{* *}$ & 0.454 & -6.17 \\
\hline Intercept & & $-1.994^{* *}$ & 0.228 & -8.75 & $-4.917^{* *}$ & 0.401 & -12.26 \\
\hline Likelihood ratio & & & $\chi^{2}(56)$ & $0.98 ; p<$ & & & \\
\hline McFadden pseudo $R^{2}$ & & & & 434 & & & \\
\hline Sample size & & & & 999 & & & \\
\hline
\end{tabular}

Note: ${ }^{* *}$ and ${ }^{*}$ indicate that variables are significant at $1 \%$ and $5 \%$ significance levels, respectively; S.E. means the standard error; - means this variable is not significant in the model.

validate and enrich existing findings but also clarify the impact of driver gender difference on SV crash severity under foggy and clear weather.

In terms of age, the marginal effect indicated that compared with middle-age drivers, the probability of SFI injury of young drivers (age $<30$ ) in SVCF is reduced by $3.2 \%$. The parameter corresponding to the SFI obeys a normal distribution with a mean of $-0.476(0.158)$ and a standard deviation of $1.10(0.106)$. However, the effect of young drivers on SVCC injury severity is not significant. This discrepancy finding is of great interest and can be used to develop management measures such as increased supervision of middle-age drivers in foggy weather.

In SVCF and SVCC, older drivers (age >60) lead to a significant increase in the probability of both slight and SFI injuries. The marginal effect indicated that the probability of SFI injury increased by $11.9 \%$ and $8.7 \%$, respectively. AbdelAty [26] reached the same conclusion that older drivers are most vulnerable to serious injuries among different age groups, as they need more reaction time in case of emergency and are more likely to sustain serious injury in case of a crash.

As can be seen from the model, no seatbelt use has a significant impact on the severity of both SVCF and SVCC crashes. Drivers not using a seat belt are $7.1 \%$ and $7.6 \%$ more likely to suffer SFI injuries in SVCF and SVCC, respectively, which is consistent with the findings of previous studies $[11,27]$. Seatbelts, as an important part of passive safety defense measures, can restrain occupant displacement and absorb impact energy in a vehicle collision or emergency braking effectively. Especially when driving in foggy weather, it is difficult for drivers to maintain a satisfactory driving condition because they cannot distinguish obstacles ahead. Seatbelt use becomes even more important to protect drivers from fatal crashes. However, $20.6 \%$ of drivers still did not use seat belts in the total sample. It is necessary for traffic managers to take measure for this phenomenon, such as more publicity and education. 
TABLE 4: Model estimation results of SVCF.

\begin{tabular}{|c|c|c|c|c|c|c|c|}
\hline \multirow{2}{*}{ Variable } & \multirow{2}{*}{ Description } & \multicolumn{3}{|c|}{ Slight injury } & \multicolumn{3}{|c|}{ SFI } \\
\hline & & Mean & S.E. & $z$-value & Mean & S.E. & $z$-value \\
\hline \multicolumn{8}{|l|}{ Driver characteristics } \\
\hline \multirow{3}{*}{ Gender } & Male & $-0.948^{* *}$ & 0.176 & -5.39 & $-1.132^{* *}$ & 0.255 & -4.44 \\
\hline & Std. dev. & - & - & - & 0.881 & 0.120 & - \\
\hline & $<30$ & - & - & - & $-0.476^{* *}$ & 0.158 & -3.01 \\
\hline \multirow[t]{2}{*}{ Age } & Std. dev. & - & - & - & 1.10 & 0.106 & - \\
\hline & $>60$ & $1.100^{* *}$ & 0.195 & 5.64 & $1.834^{* *}$ & 0.225 & 8.15 \\
\hline Seat belt used & Not used & $0.262^{*}$ & 0.109 & 2.40 & $1.017^{* *}$ & 0.134 & 7.59 \\
\hline Alcohol-impaired & Yes & $0.619^{* *}$ & 0.129 & 4.80 & $2.407^{* *}$ & 0.140 & 17.19 \\
\hline Career & Farmer & - & - & - & $0.487^{*}$ & 0.230 & 2.12 \\
\hline Driving license & With license & - & - & - & $-0.538^{* *}$ & 0.183 & -2.94 \\
\hline \multicolumn{8}{|l|}{ Vehicle characteristics } \\
\hline \multirow{4}{*}{ Vehicle type } & Motorcycle & $2.884^{* *}$ & 0.115 & 25.08 & $3.289^{* *}$ & 0.179 & 18.37 \\
\hline & Pickup & $-0.721^{* *}$ & 0.167 & -4.32 & $-0.983^{* *}$ & 0.277 & -3.55 \\
\hline & Truck & - & - & - & $-0.619^{* *}$ & 0.194 & -3.19 \\
\hline & Std. dev. & - & - & - & 0.650 & 0.116 & - \\
\hline \multicolumn{8}{|l|}{ Road characteristics } \\
\hline Road surface & Non-dry & - & - & - & $-0.593^{* *}$ & 0.187 & -3.17 \\
\hline Traffic controls & Signal control & $0.543^{* *}$ & 0.206 & 2.64 & $0.603^{*}$ & 0.289 & 2.09 \\
\hline \multicolumn{8}{|l|}{ Other characteristics } \\
\hline \multirow{2}{*}{ Area } & Rural & - & - & - & $0.234^{*}$ & 0.108 & 2.17 \\
\hline & $07: 00-09: 00$ & $-0.593^{* *}$ & 0.210 & -2.82 & - & - & - \\
\hline \multirow[t]{2}{*}{ Time of accident } & $20: 00-24: 00$ & $0.388^{* *}$ & 0.137 & 2.83 & $0.581^{* *}$ & 0.186 & 3.12 \\
\hline & $00: 00-07: 00$ & $0.292^{*}$ & 0.145 & 2.01 & $1.075^{* *}$ & 0.187 & 5.75 \\
\hline \multirow{2}{*}{ Crash type } & Fixed object & $1.609^{* *}$ & 0.197 & 8.17 & $3.053^{* *}$ & 0.206 & 14.82 \\
\hline & Pedestrian & $-1.718^{* *}$ & 0.129 & 13.32 & $-2.358^{* *}$ & 0.218 & -10.82 \\
\hline Intercept & & $-1.831^{* *}$ & 0.266 & -6.89 & $-3.480^{* *}$ & 0.346 & -10.06 \\
\hline Likelihood ratio & & & $\chi^{2}(48)$ & $8.21 ; p<$ & & & \\
\hline McFadden pseudo $R^{2}$ & & & & 348 & & & \\
\hline Sample size & & & & 138 & & & \\
\hline
\end{tabular}

Note: ${ }^{* *}$ and ${ }^{*}$ indicate that variables are significant at $1 \%$ and $5 \%$ significance levels, respectively; S.E. means the standard error; - means this variable is not significant in the model.

Driver alcohol-impaired has a significant effect on the severity of SVCF and SVCC, and the probability of SFI increases by $15.5 \%$ and $12.9 \%$, respectively. This finding can be confirmed in the existing literature. For example, it was found that the probability of fatal crashes caused by drunk driving increased by $150 \%$ [28]. This finding makes sense because alcohol can affect a driver's psychological and physiological functions (such as reaction time, vision, and agility). Also, it is difficult to maintain a normal driving state in case of emergency, which leads to an increased probability of serious crashes. Increasing driver education and enforcement intensity are effective measures to reduce driving under the influence (DUI). In addition, advanced vehicle identification systems can be developed to automatically monitor a driver's driving status and alcohol concentration in real time. If national standards are not met, mandatory measures could be implemented.

Significant differences are shown in the effect of occupation factors on the severity of SVCF and SVCC. Compared with company employment, the probability of SFI caused by self-employment decreases by $2.1 \%$ in SVCC, but this variable has no significant impact on injury severity of SVCF. On the contrary, the coefficient of farmers corresponding to SFI injury is the opposite in the SVCC and SVCF models (the marginal effects of SVCC and SVCF are -0.015 and 0.036 , respectively). All valuable findings suggest that there are significant differences in the influencing factors and effects of the severity of SVCC and SVCF. The influence of driver occupation on the severity of SV crashes in foggy weather is well revealed.

Model results indicate that with/without driver license is significant at a $90 \%$ confidence level $(p<0.01)$. The probability of SFI in SVCF and SVCC with driving license is 3\% and $4.9 \%$ lower than that without driving license, respectively [29]. This finding suggests that traffic managers should strengthen management of driver licensing.

4.2. Vehicle Characteristics. Compared with passenger cars, the probability of SFI of motorcycles in SVCF and SVCC accidents increased by $14.4 \%$ and $4.7 \%$, respectively, which is consistent with the existing literature [22]. Motorcycles are limited by their own characteristics and cannot provide adequate protection for a driver in a crash, which makes the driver exposed and easy to cause SFI. The rollover phenomenon tends to occur in motorcycle SV crashes, which increases the probability of SFI. In detail, it can be seen from the marginal effect that the probability of motorcycle drivers 
TABLE 5: Average marginal effects of significant variables.

\begin{tabular}{|c|c|c|c|c|c|}
\hline \multirow{2}{*}{ Variable } & \multirow{2}{*}{ Description } & \multicolumn{2}{|c|}{ SVCC } & \multicolumn{2}{|c|}{ SVCF } \\
\hline & & Slight injury & SFI & Slight injury & SFI \\
\hline \multicolumn{6}{|c|}{ Driver characteristics } \\
\hline Gender & Male & -0.061 & -0.003 & -0.081 & -0.045 \\
\hline \multirow{2}{*}{ Age } & $<30$ & - & - & - & -0.032 \\
\hline & $>60$ & 0.083 & 0.087 & 0.067 & 0.119 \\
\hline \multirow{2}{*}{$\begin{array}{l}\text { Seat belt used } \\
\text { Alcohol-impaired }\end{array}$} & Not used & -0.012 & 0.076 & -0.010 & 0.071 \\
\hline & Yes & 0.008 & 0.129 & -0.016 & 0.155 \\
\hline \multirow{2}{*}{ Career } & Self-employed & - & -0.021 & - & - \\
\hline & Farmer & - & -0.015 & - & 0.036 \\
\hline Driving license & With license & -0.049 & -0.018 & - & -0.030 \\
\hline \multicolumn{6}{|c|}{ Vehicle characteristics } \\
\hline \multirow{3}{*}{ Vehicle type } & Motorcycle & 0.341 & 0.047 & 0.356 & 0.144 \\
\hline & Pickup & -0.054 & -0.015 & -0.067 & -0.043 \\
\hline & Truck & - & -0.029 & - & -0.037 \\
\hline \multicolumn{6}{|c|}{ Road characteristics } \\
\hline Road surface & Non-dry & - & - & - & -0.032 \\
\hline Traffic controls & Signal control & - & - & 0.048 & 0.026 \\
\hline \multicolumn{6}{|c|}{ Other characteristics } \\
\hline Area & Rural & 0.023 & 0.011 & - & 0.014 \\
\hline \multirow[t]{2}{*}{ Week } & Weekend & - & 0.023 & - & - \\
\hline & $07: 00-09: 00$ & -0.031 & - & -0.054 & - \\
\hline \multirow[t]{2}{*}{ Time of accident } & $20: 00-24: 00$ & 0.091 & 0.036 & 0.031 & 0.024 \\
\hline & $00: 00-07: 00$ & - & 0.041 & -0.008 & 0.074 \\
\hline \multirow{2}{*}{ Crash type } & Fixed object & 0.107 & 0.201 & 0.061 & 0.266 \\
\hline & Pedestrian & -0.114 & -0.052 & -0.133 & -0.085 \\
\hline
\end{tabular}

Note: - means this variable is not significant in the model.

suffering from SFI in SVCF is significantly higher than that in SVCC (in the SVCF and SVCC models, the marginal effects of motorcycle drivers corresponding to SFI were 0.144 and 0.047 , respectively). This may be due to low visibility in foggy weather, which affects a driver's correct judgment of headway distance.

In the SVCF and SVCC models, pickup and a driver's probability of slight injury and SFI injury are significantly negatively correlated (using passenger car as the reference). The reason is that the driving vision of a pickup is better than that of a passenger car and it can absorb more energy in crashes so as to reduce the risk of SFI. Furthermore, Desapriya et al. [30] found that when a passenger car collided with a pickup, the injury to the pickup driver was significantly lower than that of the passenger car driver. This finding confirms the validity of the conclusions drawn in this study.

Compared to passenger cars, the probability of SFI in truck-related SVCF and SVCC crashes are reduced by $3.7 \%$ and $2.9 \%$, respectively. A similar conclusion was reached by Bédard et al. [31]. More specifically, the parameters of a truck corresponding to SFI in the SVCF model obey the normal distribution, with mean value of -0.619 and standard deviation of 0.650 . However, there is no heterogeneity for this variable in the SVCC model. By constructing the MLMs under the conditions of SVCF and SVCC, respectively, the heterogeneity of influencing factors in the subset can be effectively identified. It is valuable for understanding the source of heterogeneity in the whole dataset.
4.3. Road Characteristics. In the SVCF model, road surface condition has a significant impact on SFI. The average marginal effect indicates that the probability of SFI caused by non-dry pavement decreases by $3.2 \%$ with a dry road surface as the reference. In foggy weather, drivers are more cautious when driving on non-dry road surface and usually keep a low speed. Thus, the probability of SFI will be reduced when an SV crash occur, but road surface condition has no significant effect on the severity of SVCC. This finding is equally contributory and can further improve the conclusions of $\mathrm{Li}$ et al. [8], who found a significant effect of the non-dry road surface on the severity of low visibility-related SV crashes and did not investigate clear weather scenarios.

The influence of signal control on the severity of SV crashes is significant on foggy days but not on sunny days. Specifically, the probability of slight injury and SFI injury caused by signal control increases by $4.8 \%$ and $2.6 \%$, respectively, compared with uncontrolled roads in the SVCF model. This may be due to low visibility in foggy weather, and fog will dilute the color depth of the signal light [15]. Drivers cannot judge the color of the signal lamp in time. Thus, it is easier to hit roadside fixed facilities such as curb and guardrail.

4.4. Other Characteristics. Compared with the urban area, the probability of SFI injury increased by $1.1 \%$ and $1.4 \%$, respectively, when SVCC and SVCF occurred in rural areas [32]. This is justified because traffic management in rural areas is unsatisfactory and speed on national highways is 
high. Thus, the probability of serious crashes in rural areas is higher than that in urban areas.

In addition, the probability of SFI injury caused by a weekend day is significantly higher than that of a working day (marginal effect is 0.023 ) in the SVCC model. This is in agreement with the findings of previous studies. For example, Abrari Vajari et al. [33] conducted modeling and analysis on motorcycle crashes and found that the probability of fatal crashes on weekend days was 1.14 times that of working days. Salum et al. [34] noted that drivers with less driving experience are more likely to drive on weekends, which further supports our findings. However, there is not statistically significant relationship between weekend and SFI crashes in the SVCF model. This discovery is interesting and meaningful. It can verify the necessity of modeling clear and foggy days separately. More importantly, the traffic management department can formulate scientific management measures based on this discovery. For example, the management of weekend under clear weather should be strengthened.

For different time periods, 7:00-9:00 (morning peak), $20: 00-24: 00$, and $00: 00-7: 00$ have a significant effect on the severity of SVCF and SVCC, but the effect varies with time. The average marginal effects indicate that compared with 9:00-17:00 in SVCF and SVCC accidents, the probability of slight injury in the morning peak is reduced by $5.4 \%$ and $3.1 \%$, respectively. This is because traffic is congested in the morning peak hours and driving speed is low. Thus, a driver is less likely to be injured in a collision. In addition, the probability of causing SFI injuries increased by $2.4 \%$ and $3.6 \%$ in SVCF and SVCC crashes from $20: 00-24$ : 00 , respectively. There is also a significant positive correlation between 0:00-7:00 and injury severity (marginal effects of SVCF and SVCC are 0.074 and 0.041, respectively), which is consistent with the findings of previous studies [33]. The reason for this phenomenon may be that traffic control during the evening hours is weak, and drivers are more likely to drive fatigued or under the influence of alcohol. Increasing the monitoring of driving behavior during evening hours is considered a necessary measure to reduce the probability of severe crashes.

In the SVCF and SVCC models, the probability of SFI injury caused by collision with a fixed object increased by $26.6 \%$ and $20.1 \%$, respectively. Other literature on this factor has reached consistent conclusions [35]. This may be due to the fact that when motor vehicles collide with roadside concrete facilities or other fixed obstacles, the collision energy cannot be absorbed by the fixed objects. Therefore, energy-absorbing devices should be installed on the surface of fixed obstacles (such as concrete facilities) to reduce the injury severity of SV crashes.

SV crashes with pedestrians are significantly and negatively correlated with driver injury severity. The marginal effects of the SVCF and SVCC models showed that drivers are $8.5 \%$ and $5.2 \%$ less likely to have a serious injury, respectively. It is reasonable to draw this conclusion because compared with motor vehicles, pedestrians are vulnerable. Collision with pedestrians will not cause serious injury to drivers but will have a serious impact on pedestrians.
TABLE 6: Model transferability test.

\begin{tabular}{lcc}
\hline Crash model & \multicolumn{2}{c}{ Application data } \\
& SVCC & SVCF \\
\hline SVCC & 1 & -0.172 \\
SVCF & -0.306 & 1 \\
\hline
\end{tabular}

4.5. Verify Model Transferability. To evaluate the model transferability under clear and foggy weather conditions, the TI values of the crash model under the two weather conditions were calculated. The specific calculation results are shown in Table 6. It is necessary to point out that the TI value of the crash severity analysis model for its local dataset is 1 because the model represents the local situation of the crash dataset. In addition, the remaining TI values in Table 6 are less than 0 . This shows that the fitting performance of the intercept-only model established by local data is better than the transferred model. Hence, the model transferability test shows that the crash model established by SVCF and SVCC cannot be directly transferred. In the process of assessing the risk factors of crash severity, it is recommended to build the SVCC model and SVCF model, respectively.

\section{Conclusion}

This study used 10,137 SV crashes in Shandong Province, China, as sample data (from 2015 to 2019) and divided the dataset into two parts for modeling: single-vehicle crashes in foggy weather (SVCF) and single-vehicle crashes in clear weather (SVCC). On this basis, the MLM was employed to analyze the factors that have significant impact on the severity of SVCF and SVCC crashes. By examining the McFadden pseudo $R$-squared and random coefficient distributions of the model, it is concluded that the goodness-offit is satisfactory and could effectively capture unobserved heterogeneity across observations. In addition, the results of the model transferability test showed that the crash models established by SVCC and SVCF cannot be directly transferred to each other. It is recommended to build the SVCC model and SVCF model separately.

The findings in this study are as follows:

(1) There are significant differences in the factors affecting injury severity of SV crashes in foggy and clear weather. In foggy weather crashes, both young drivers and non-dry road surfaces resulted in a 3.2\% reduction in the probability of SFI. Signal control resulted in a $2.6 \%$ increase in the probability of SFI in SVCF. However, none of these variables have a significant effect on clear weather crashes. The probability of self-employment leading to SFI in clear weather crashes is reduced by $2.1 \%$. Weekends resulted in a $2.3 \%$ increase in the probability of SFI in SVCC. However, two variables of foggy crashes are not significant. Interestingly, the effect of the farmer variable on the severity of foggy and clear weather crashes showed an opposite trend, i.e., the variable resulted in a $1.5 \%$ decrease in the probability of SFI 
injuries in clear weather crashes and a 3.6\% increase in the probability of SFI injuries in foggy weather crashes.

(2) There is a commonality in the factors influencing the severity of SV crashes in foggy and clear weather. For instance, male drivers, having a license and using a pickup or a truck, resulted in significantly lower severity of both foggy and clear weather crashes. Older drivers, seat belts not used, drunk driving, motorcycles, rural areas, night time (20:00-07:00), and collisions with fixed objects resulted in significantly higher crash severity in both foggy and clear weather.

Targeted policies based on the conclusions are more cost-effective in reducing the severity of crashes. For example, in both foggy and clear weather, driving violations resulted in a significant increase in crash severity. Hence, advance traffic crash warning systems should be installed to detect dangerous driving behaviors (such as drunk driving and seat belts not used). Vehicle-to-road communication (V2I) technology can be used to provide road information in real time [36].

Collisions with fixed objects increase driver injury severity significantly. Thus, the focus should be on improvement of hard guardrails or other road surface fixtures on both sides of the road. The Department of Transportation should consider replacing hard guardrails with soft guardrails that can absorb collision energy; an energy-absorbing buffer device is installed on the fixed surface to reduce the severity of collision.

In the future, clustering techniques will be used to classify the data and reduce the heterogeneity of the data.

\section{Data Availability}

The traffic crash data used to support the findings of this study have not been made available because traffic accident data are required to be confidential by Shandong Department of Transportation.

\section{Conflicts of Interest}

The authors declare that there are no conflicts of interest regarding the publication of this paper.

\section{Acknowledgments}

The authors would like to thank the support of the Shandong Department of Transportation (SDOT) and Zibo Department of Transportation (ZDOT). This research was jointly supported by the National Natural Science Foundation of China (Grant nos. 71901134 and 71871057), National Science Foundation for Distinguished Young Scholars (Grant no. 51925801), Natural Science Foundation of Shandong (Grant no. ZR2018BF024), and Postdoctoral Research Assistance Program of Jiangsu (Grant no. 2018K118C).

\section{References}

[1] National Highway Traffic Safety Administration (NHTSA), Traffic Safety Facts Annual Report Tables, National Highway Traffic Safety Administration, Washington, DC, USA, 2018.

[2] M. Zhou and H. C. Chin, "Factors affecting the injury severity of out-of-control single-vehicle crashes in Singapore," Accident Analysis \& Prevention, vol. 124, pp. 104-112, 2019.

[3] S. M. Rifaat and H. C. Chin, "Accident severity analysis using ordered probit model," Journal of Advanced Transportation, vol. 41, no. 1, pp. 91-114, 2007.

[4] D. Ma, X. Luo, S. Jin, D. Wang, W. Guo, and F. Wang, "Lanebased saturation degree estimation for signalized intersections using travel time data," IEEE Intelligent Transportation Systems Magazine, vol. 9, no. 3, pp. 136-148, 2017.

[5] D. Ma, X. Luo, W. Li, S. Jin, W. Guo, and D. Wang, "Traffic demand estimation for lane groups at signal-controlled intersections using travel times from video-imaging detectors," IET Intelligent Transport Systems, vol. 11, no. 4, pp. 222-229, 2017.

[6] C. Xu, W. Wang, and P. Liu, "Identifying crash-prone traffic conditions under different weather on freeways," Journal of Safety Research, vol. 46, pp. 135-144, 2013.

[7] Y. Wu, M. Abdel-Aty, and J. Lee, "Crash risk analysis during fog conditions using real-time traffic data," Accident Analysis \& Prevention, vol. 114, pp. 4-11, 2018.

[8] Z. Li, C. Chen, Q. Wu et al., "Exploring driver injury severity patterns and causes in low visibility related single-vehicle crashes using a finite mixture random parameters model," Analytic Methods in Accident Research, vol. 20, pp. 1-14, 2018.

[9] M. Feng, X. Wang, J. Lee et al., "Transferability of safety performance functions and hotspot identification for freeways of the United States and China," Accident Analysis \& Prevention, vol. 139, p. 105493, 2020.

[10] H. Wen and G. Xue, "Injury severity analysis of familiar drivers and unfamiliar drivers in single-vehicle crashes on the mountainous highways," Accident Analysis \& Prevention, vol. 144, p. 105667, 2020.

[11] Z. Li, Y. Ci, C. Chen et al., "Investigation of driver injury severities in rural single-vehicle crashes under rain conditions using mixed logit and latent class models," Accident Analysis \& Prevention, vol. 124, pp. 219-229, 2019.

[12] H. Yu, R. Yuan, Z. Li et al., "Identifying heterogeneous factors for driver injury severity variations in snow-related rural single-vehicle crashes," Accident Analysis \& Prevention, vol. 144, p. 105587, 2020.

[13] R. L. Moore and L. Cooper, "Fog and road traffic," TRRL Report, Transport and Road Research Laboratory, Wokingham, UK, 1972.

[14] M. Abdel-Aty, A.-A. Ekram, H. Huang, and K. Choi, "A study on crashes related to visibility obstruction due to fog and smoke," Accident Analysis \& Prevention, vol. 43, no. 5, pp. 1730-1737, 2011.

[15] J.-P. Tarel, N. Hautiere, L. Caraffa, A. Cord, H. Halmaoui, and D. Gruyer, "Vision enhancement in homogeneous and heterogeneous fog," IEEE Intelligent Transportation Systems Magazine, vol. 4, no. 2, pp. 6-20, 2012.

[16] Y. Guo, Z. Li, P. Liu, and Y. Wu, "Modeling correlation and heterogeneity in crash rates by collision types using full Bayesian random parameters multivariate tobit model," Accident Analysis \& Prevention, vol. 128, pp. 164-174, 2019.

[17] J. Lee and F. Mannering, "Impact of roadside features on the frequency and severity of run-off-roadway accidents: an 
empirical analysis," Accident Analysis \& Prevention, vol. 34, no. 2, pp. 149-161, 2002.

[18] F. L. Mannering, V. Shankar, C. R. Bhat et al., "Unobserved heterogeneity and the statistical analysis of highway accident data," Analytic Methods in Accident Research, vol. 11, pp. 1-16, 2016.

[19] F. Ye and D. Lord, "Comparing three commonly used crash severity models on sample size requirements: multinomial logit, ordered probit and mixed logit models," Analytic Methods in Accident Research, vol. 1, pp. 72-85, 2014.

[20] H. Xiong, X. Zhu, and R. Zhang, "Energy recovery strategy numerical simulation for dual axle drive pure electric vehicle based on motor loss model and big data calculation," Complexity, vol. 2018, Article ID 4071743, 14 pages, 2018.

[21] Y. Guo, T. Sayed, L. Zheng et al., "A hierarchical Bayesian peak over threshold approach for conflict-based before-after safety evaluation of leading pedestrian intervals," Accident Analysis \& Prevention, vol. 147, p. 105772, 2020.

[22] Z. Li, P. Liu, W. Wang, and C. Xu, "Using support vector machine models for crash injury severity analysis," Accident Analysis \& Prevention, vol. 45, pp. 478-486, 2012.

[23] R. O. Mujalli and J. de Oña, "Injury severity models for motor vehicle accidents: a review," Proceedings of the Institution of Civil Engineers-Transport, vol. 166, no. 5, pp. 255-270, 2013.

[24] D. Ma, X. Luo, S. Jin, W. Guo, and D. Wang, "Estimating maximum queue length for traffic lane groups using travel times from video-imaging data," IEEE Intelligent Transportation Systems Magazine, vol. 10, no. 3, pp. 123-134, 2018.

[25] Y. Guo, Z. Li, Y. Wu, and C. Xu, "Exploring unobserved heterogeneity in bicyclists' red-light running behaviors at different crossing facilities," Accident Analysis \& Prevention, vol. 115, pp. 118-127, 2018.

[26] M. Abdel-Aty, "Analysis of driver injury severity levels at multiple locations using ordered probit models," Journal of Safety Research, vol. 34, no. 5, pp. 597-603, 2003.

[27] D. Ma, X. Song, and P. Li, "Daily traffic flow forecasting through a contextual convolutional recurrent neural network modeling inter-and intra-day traffic patterns," IEEE Transactions on Intelligent Transportation Systems, vol. 99, pp. 2627-2636, 2020.

[28] J.-K. Kim, G. F. Ulfarsson, S. Kim, and V. N. Shankar, "Driverinjury severity in single-vehicle crashes in California: a mixed logit analysis of heterogeneity due to age and gender," Accident Analysis \& Prevention, vol. 50, pp. 1073-1081, 2013.

[29] Y. Guo, Z. Li, P. Liu, and Y. Wu, "Exploring risk factors with crashes by collision type at freeway diverge areas: accounting for unobserved heterogeneity," IEEE Access, vol. 7, pp. 11809-11819, 2019.

[30] E. Desapriya, I. Pike, and J. Kinney, "The risk of injury and vehicle damage severity in vehicle mismatched side impact crashes in British Columbia," IATSS Research, vol. 29, no. 2, pp. 60-66, 2005.

[31] M. Bédard, G. H. Guyatt, M. J. Stones, and J. P. Hirdes, "The independent contribution of driver, crash, and vehicle characteristics to driver fatalities," Accident Analysis \& Prevention, vol. 34, no. 6, pp. 717-727, 2002.

[32] S. Yasmin, N. Eluru, C. R. Bhat, and R. Tay, "A latent segmentation based generalized ordered logit model to examine factors influencing driver injury severity," Analytic Methods in Accident Research, vol. 1, pp. 23-38, 2014.

[33] M. Abrari Vajari, K. Aghabayk, M. Sadeghian, and N. Shiwakoti, "A multinomial logit model of motorcycle crash severity at Australian intersections," Journal of Safety Research, vol. 73, pp. 17-24, 2020.
[34] J. H. Salum, A. E. Kitali, H. Bwire, T. Sando, and P. Alluri, "Severity of motorcycle crashes in Dares Salaam, Tanzania," Traffic Injury Prevention, vol. 20, no. 2, pp. 189-195, 2019.

[35] X. Zhu and S. Srinivasan, "A comprehensive analysis of factors influencing the injury severity of large-truck crashes," Accident Analysis \& Prevention, vol. 43, no. 1, pp. 49-57, 2011.

[36] R.-H. Zhang, Z.-C. He, H.-W. Wang, F. You, and K.-N. Li, "Study on self-tuning tyre friction control for developing main-servo loop integrated chassis control system," IEEE Access, vol. 5, pp. 6649-6660, 2017. 